\title{
SIMULACIÓN ANALÍTICA DEL COMPORTAMIENTO NO LINEAL DE MUROS DIAFRAGMA DE MAMPOSTERÍA SUJETOS A CARGAS LATERALES.
}

Agustín Orduña ${ }^{(1)}$ y A. Gustavo Ayala(2)

\section{RESUMEN}

En este artículo se presenta un modelo no lineal de elementos finitos de interés en el análisis de muros de relleno de mampostería en marcos de concreto reforzado sometidos a cargas laterales no cíclicas, se describen brevemente los modelos constitutivos utilizados para los materiales. La validez del modelo se comprueba mediante un ejemplo ilustrativo cuyos resultados reproducen satisfactoriamente a los experimentales. Como aplicación práctica de este estudio se dan las bases para el desarrollo de una herramienta simplificada de análisis de marcos rellenos, útil en el diseño sísmico de edificios. El modelo simplificado se basa en sustituir al muro diafragma por tres barras biarticuladas en compresión, con comportamiento elasto-plástico perfecto.

\footnotetext{
Artículo recibido el 23 de febrero de 2001 y aprobado para su publicación el 11 de mayo de 2001. Se aceptarán comentarios y/o discusiones al artículo hasta cinco meses después de su publicación.

${ }^{1}$ Instituto de Ingeniería, Universidad Nacional Autónoma de México. Ciudad Universitaria. Apdo. postal 70-642, Coyoacán, CP 04510. México, D.F. México tel. (525)6223466, fax (525)6161514
} 


\section{INTRODUCCIÓN}

Las tendencias actuales de diseño sísmico de estructuras basado en desempeño, requieren conocer con detalle el comportamiento no lineal de las estructuras desde el inicio de éste, hasta niveles altos de deformación lateral. Los métodos de análisis elástico lineales, en general, no proporcionan la información necesaria para el diseño, por lo que deben ser sustituidos por técnicas y programas de análisis no lineal. Sin embargo, los ingenieros de la práctica requieren de modelos no lineales de elementos estructurales que sean suficientemente simples para su uso cotidiano, y que, al mismo tiempo, representen en forma realista su comportamiento.

El comportamiento de estructuras de marcos es mejor entendido que el de otros sistemas estructurales, ya que la mecánica de materiales ha proporcionado, en los elementos barra, una herramienta suficientemente precisa para su análisis, tanto para los intervalos lineal como no lineal. El problema se complica, y mucho, cuando se incluyen muros en las crujías de los marcos, debido a que no existe un modelo simple, como en el caso de las barras, que represente el comportamiento de estos elementos y su relación con los que lo confinan. El problema se torna aún más complejo cuando el muro es de mampostería, ya que el comportamiento de este material es mucho menos conocido que el del acero o el del concreto. Esto es así a pesar de que la mampostería es uno de los materiales constructivos más antiguos, con más de diez mil años de historia, y a pesar de que desde hace más de 40 años se realizan investigaciones de calidad sobre su comportamiento. Entre las razones principales del desconocimiento de su comportamiento se pueden mencionar: la complejidad de su comportamiento mecánico; el gran número de variables que es necesario tomar en cuenta para su estudio; y que algunas de ellas, como las propiedades de los materiales, la calidad de la mano de obra, las condiciones de contacto entre el muro y el marco o la adherencia entre mortero y piezas, son muy difíciles de controlar y/o cuantificar.

Es costumbre común en el diseño de edificios despreciar la contribución de los muros de relleno. Se argumenta que si el marco está diseñado para resistir las acciones laterales a las que estará sujeto durante su vida útil, la presencia de los muros es una reserva de resistencia. Además, es común que estos muros sean colocados y retirados de acuerdo con las diferentes necesidades de los ocupantes del inmueble durante su vida útil. Sin embargo, la presencia de muros de relleno debe considerarse en el diseño de una estructura, ya que se ha comprobado que modifican drásticamente el comportamiento sísmico de marcos respecto al que se observaría en su ausencia. Los muros de relleno incrementan sustancialmente la rigidez de los marcos, con lo que se reducen los períodos naturales de vibración, y se modifican las acciones sísmicas a las que estará sujeto. Estos muros también incrementan significativamente la resistencia a cargas laterales de la estructura, pueden contribuir a la disipación de energía durante un sismo, y aunque un muro de mampostería simple se comporta de manera frágil, con un buen detallado del marco se pueden 
obtener comportamientos dúctiles de la estructura. La interacción entre muro y marco a lo largo de las zonas de contacto modifica tanto la magnitud como la distribución de los elementos mecánicos en trabes y columnas, al ignorar la presencia de los muros algunas secciones pueden quedar sobradas en resistencia, y otras escasas. Sin embargo, este aspecto no ha sido estudiado con detalle, la mayoría de las investigaciones se han concentrado en el comportamiento global de este tipo de estructuras. Los muros de relleno usados con un buen criterio ingenieril pueden mejorar sustancialmente el comportamiento sísmico de edificios; sin embargo, cuando se usan en distribuciones irregulares en planta o elevación, pueden llegar a ser la causa del colapso de estructuras, por efectos de torsión o cambios bruscos de rigidez con la altura. También pueden producir daño cuando son ignorados en el diseño, aún cuando estén distribuidos regularmente.

El problema de interacción marco-muro de relleno está aún lejos de ser resuelto. Existe la necesidad de contar con más resultados experimentales que difícilmente podrá obtenerse dado el alto costo involucrado en este tipo de estudios. Una salida alterna a este problema es desarrollar procedimientos analíticos que permitan simular estos experimentos, y a partir de ellos proponer modelos de análisis menos elaborados para su uso en aplicaciones cotidianas de la ingeniería estructural. Estos modelos deben nutrirse de información experimental adecuada con sus necesidades, relativa a las propiedades mecánicas de los materiales, y deben validarse con resultados experimentales en modelos completos. Para lograr lo anterior es necesario incrementar la comunicación entre experimentalistas y analistas.

El desarrollo que ha tenido la computación en las últimas décadas ha permitido la aplicación del Método de los Elementos Finitos (MEF) al análisis de estructuras de formas y comportamientos complejos. Por ello es que el MEF constituye actualmente una de las herramientas fundamentales para el estudio del comportamiento de elementos y estructuras de mampostería; principalmente en el ámbito de la investigación, ya que la gran cantidad de recursos que requiere un análisis no lineal con elementos finitos hace que su uso sea inadecuado para la práctica común de la ingeniería. Lo anterior ha sido reconocido por diversos investigadores quienes han realizado estudios con diferentes grados de refinamiento mediante el uso del MEF. Desgraciadamente, la mayoría de estos estudios se ha orientado a la solución de problemas elásticos, y en algunos casos los efectos no lineales se consideran de una manera gruesamente aproximada. Estos estudios han dado lugar a elementos estructurales equivalentes a los muros que, si bien permiten incluir su efecto en el comportamiento global de la estructura, no permiten conocer su comportamiento local aún en el intervalo lineal.

Para estudiar el comportamiento de muros diafragma en marcos de concreto reforzado en este trabajo se discuten los aspectos que se consideran más importantes del comportamiento de muros diafragma de mampostería. Se desarrolla un modelo no lineal de elementos finitos de un 
marco de un nivel y una crujía con un muro diafragma de mampostería sin huecos. Se describen sus características y se comentan algunas experiencias adquiridas durante el proceso de solución y de calibración del mismo. Finalmente, con base en la información obtenida del análisis, se propone un modelo simplificado, consistente en tres elementos diagonales, para el análisis de marcos con muros de relleno sujetos a cargas sísmicas.

\section{COMPORTAMIENTO DE MUROS DIAFRAGMA DE MAMPOSTERÍA}

La mampostería es un material de construcción elaborado con piezas, que pueden ser de distintos materiales y tipos, unidas entre sí por medio de juntas de mortero. Las piezas y el mortero tienen propiedades mecánicas distintas, además, las superfícies de unión entre ellos presentan características que influyen de una manera muy importante en el comportamiento mecánico del conjunto. Por ello la mampostería es un material no homogéneo, aunque para muros que contienen a un gran número de piezas, y desde un punto de vista macroscópico, se puede considerar como homogénea. La forma rectangular prismática de las piezas, su colocación en patrones generalmente regulares, y la presencia de las juntas de mortero que actúan como planos de debilidad en direcciones bien definidas, sugieren que la mampostería es un material ortótropo con direcciones principales normal y paralela a las juntas horizontales.

El comportamiento no lineal de la mampostería como material proviene de dos fuentes principales. La primera es el comportamiento no lineal que presentan todos los materiales a partir de cierto nivel de deformaciones, esta característica se hereda a los elementos estructurales y a las estructuras. En este caso, los comportamientos no lineales de las piezas y del mortero, son cualitativamente similares a los del concreto: en tensión uniaxial se tiene una resistencia muy baja, comportamiento prácticamente lineal hasta el esfuerzo máximo y falla frágil o casi frágil. En compresión uniaxial la resistencia es mucho mayor, el comportamiento es marcadamente no lineal con esfuerzos crecientes con la deformación hasta un máximo, y que luego disminuyen hasta un valor residual constante. Sin embargo, cuantitativamente las propiedades mecánicas tanto lineales como no lineales de las piezas, pueden ser muy distintas a las del mortero. La segunda fuente de no linealidad es el comportamiento de la superficie de unión entre piezas y mortero, en donde ocurre agrietamiento por tensión (o separación) y deslizamiento debido a esfuerzos tangenciales. En muros que fallan por cortante este aspecto determina en forma muy significativa el comportamiento global del elemento estructural.

El comportamiento de muros no confinados ni reforzados ante la acción de cargas laterales depende fundamentalmente del nivel de carga vertical. Cuando la carga vertical es baja o nula, ocurre agrietamiento en las juntas de la parte inferior debido a los esfuerzos de tensión 
producidos por el momento flexionante, y conduce a una falla frágil del muro. Cuando se tiene una carga axial mayor, el agrietamiento se mueve hacia la diagonal en compresión, debido a la tensión diagonal que produce el cortante, y se presenta en forma de escalera a lo largo de las juntas horizontales y verticales. Al aumentar aún más la carga vertical, el agrietamiento diagonal empieza a afectar tanto a las juntas como a las piezas. Y a niveles todavía mayores de carga vertical, se presenta aplastamiento en las esquinas en compresión antes que el agrietamiento diagonal. Los niveles de carga vertical en los que ocurre la transición entre cada tipo de falla, dependen de las características mecánicas del mortero, las piezas y la interfaz entre ellos; por ejemplo, con un mortero o interfaz muy débil y piezas resistentes, la carga vertical necesaria para que fallen las piezas debe ser muy grande, en comparación de la que se requeriría en una mampostería con mortero e interfaces resistentes y piezas débiles.

En muros diafragma sujetos a carga horizontal, la carga vertical sobre el muro se va incrementando conforme aumenta la primera debido precisamente al efecto confinante del marco. La distribución de esfuerzos de contacto entre el muro y el marco depende de la rigidez relativa entre éstos, y de la relación de aspecto del muro. Por lo que, dependiendo de estos factores, la falla del muro puede estar entre el agrietamiento diagonal y el aplastamiento de las esquinas cargadas. Cuando la relación altura a ancho es grande y con ella la pendiente de la diagonal en compresión, se desarrolla una fuerza vertical alta sobre el muro, por ello son comunes en estos casos las fallas por aplastamiento en las esquinas o por agrietamiento diagonal que incluye a las piezas. Con relaciones altura a ancho pequeñas, las fuerzas horizontales se transmiten principalmente por cortante con fuerzas verticales pequeñas sobre el muro, en estos casos son comunes las fallas por deslizamiento a través de las juntas.

Adicionalmente a los comportamientos no lineales de la mampostería como material, descritos arriba, los muros diafragma presentan un comportamiento no lineal ante cargas laterales debido a efectos geométricos que se producen en la interfaz entre el muro y el marco que lo confina. Existen dos casos, el primero cuando se tiene una separación inicial entre el muro y el marco, producto de la contracción de la mampostería, o de una holgura insuficiente cuando se intenta aislar al muro del marco. En este caso inicialmente trabaja solamente el marco, hasta que su desplazamiento es suficiente para cerrar la abertura inicial, entonces empieza a tener contacto con el muro y se inicia el trabajo en conjunto. Este proceso de cerramiento es gradual conforme aumenta la carga lateral, pero, en general, no llega a haber contacto en todo el perímetro del muro. El segundo caso se presenta cuando inicialmente hay contacto entre muro y marco, pero al aplicar una carga lateral, los distintos patrones de deformación de ambos elementos producen que se empiecen a separar en las esquinas en tensión, debido a la poca o nula resistencia de la interfaz ante estas acciones. Cuando existe una pequeña separación entre muro y marco la resistencia máxima es prácticamente la misma que cuando no existe tal separación, aunque la carga de 
agrietamiento puede reducirse ligeramente (Moghaddam y Dowling, 1987). En ambos casos se presenta deslizamiento entre las superficies de contacto, que se debe también a los diferentes patrones de desplazamiento del muro y el marco. Es importante evaluar la magnitud de los esfuerzos cortantes que aparecen por efecto de la fricción entre ambas superficies ya que modifican sustancialmente el comportamiento del conjunto (Combescure et al, 1995).

El comportamiento ante cargas laterales de marcos de concreto o de acero con muros diafragma de mampostería, se puede describir en forma muy general, por medio de las siguientes etapas o modos de falla (Moghaddam y Dowling, 1987), fig 1:

i. Separación: A niveles bajos de carga ocurre separación entre el muro y el marco en las esquinas alejadas de la diagonal en compresión, esto sucede en todos los casos.

ii. Aplastamiento de esquina: En el caso en que el marco sea muy flexible, los esfuerzos de compresión en la esquina cargada se concentran en una superficie pequeña y ocurre aplastamiento de la mampostería en esa zona. A medida que el marco sea más rígido, y pueda por lo tanto distribuir mejor los esfuerzos, la zona de aplastamiento se extiende hacia el centro del muro.

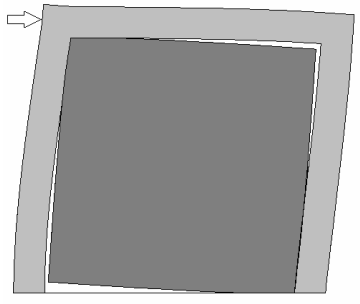

a)

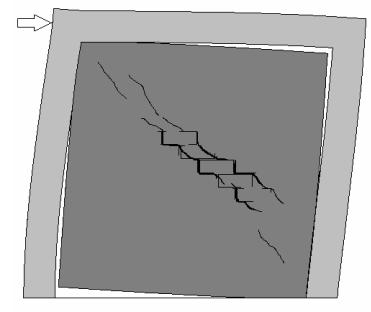

c)

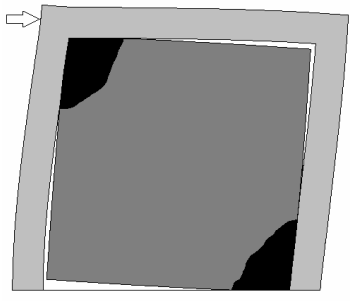

b)

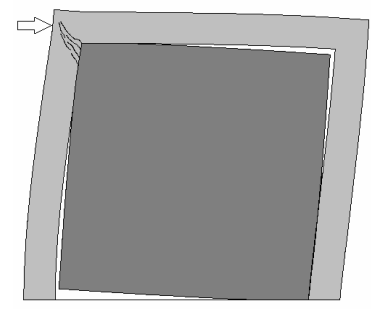

d)

Figura 1. Modos de falla de marcos con muros de relleno de mampostería (Moghaddam y Dowling, 1987); a) separación; b) aplastamiento de esquina; c) agrietamiento inclinado; d) falla de la columna en tensión. 
iii. Agrietamiento inclinado: Si el marco es suficientemente rígido para evitar el aplastamiento del muro, entonces ocurre agrietamiento a lo largo de la diagonal en compresión, a causa de los esfuerzos principales de tensión en dirección perpendicular. Este comportamiento no implica el colapso y en muchos casos se pueden alcanzar niveles de carga significativamente mayores que el correspondiente al agrietamiento.

iv. Falla de la columna en tensión: La combinación de esfuerzos de tensión y de cortante en la columna izquierda de la figura, puede producir la falla de ésta, principalmente si es de concreto y no tiene el suficiente refuerzo transversal y/o longitudinal. Es menos común que falle la columna derecha porque la compresión incrementa, hasta cierto nivel, su resistencia al cortante.

Mehrabi et al (1994) presentan un desglose más detallado de los posibles mecanismos de falla de marcos con muros de relleno, fig 2. Ellos clasifican a los tipos de falla en cinco grupos: falla por flexión (A), agrietamiento a media altura (B), agrietamiento inclinado (C), deslizamiento en las juntas horizontales (D) y aplastamiento de esquina (E).

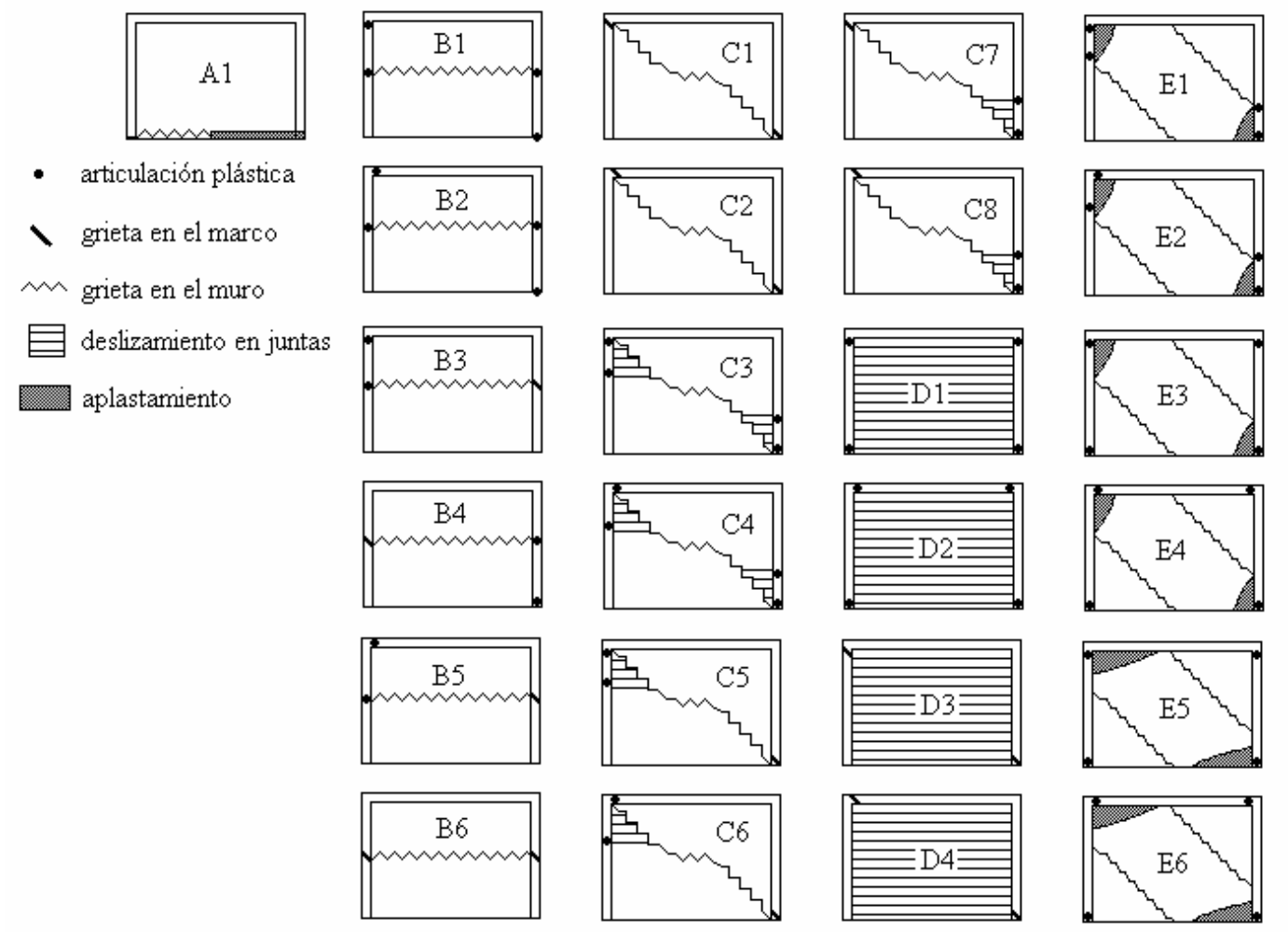

Figura 2 Mecanismos de falla de marcos con muros de relleno (Mehrabi et al. 1994). Tipos de falla posibles: falla por flexión (A), agrietamiento a media altura (B), agrietamiento inclinado (C), deslizamiento en las juntas horizontales (D) y aplastamiento de esquina (E) 
Resultados experimentales obtenidos por varios investigadores en el Instituto de Ingeniería de la UNAM y resumidos por Meli (1979), indican que las características del marco confinante no tienen una influencia significativa en el comportamiento del muro antes de la aparición del agrietamiento diagonal. Sin embargo, la reserva de resistencia y la ductilidad dependen especialmente de la resistencia a fuerzas cortantes en las esquinas del marco; ya que si ésta resistencia es baja, la grieta diagonal se prolonga en el marco y la capacidad de carga ya no aumenta significativamente, mientras que si la esquina del marco es resistente, la carga puede aumentar apreciablemente hasta que se produzca la falla por aplastamiento de la mampostería.

\section{MODELO NUMÉRICO}

Para estudiar de manera analítica el comportamiento de muros de mampostería confinados con marcos de concreto, se elaboró un modelo no lineal de elementos finitos de un marco de concreto reforzado de una crujía y un nivel con un muro de mampostería sin huecos. Para el modelo se utilizó el código DIANA (TNO 1996). Tanto el marco de concreto como el muro de mampostería se modelaron con elementos cuadriláteros de cuatro nudos en esfuerzo plano con integración gaussiana de $2 \times 2$. Alrededor del muro se introdujeron elementos interfaz de cuatro nudos para unirlo con el marco y con la superficie inferior que representa a la viga de cimentación, la cual se supone infinitamente rígida. Estos elementos interfaz modelan la posible separación y el posible deslizamiento en el perímetro del muro, para su integración numérica se utilizó una cuadratura de concentración (cuadratura de Lobbato), ya que se ha comprobado que es más adecuada en este tipo de elementos que una integración continua (TNO 1996). El acero de refuerzo del marco se modeló con un recurso disponible en DIANA que consiste en agregar la rigidez del refuerzo a la del elemento que representa al concreto. Las barras longitudinales se modelaron en forma discreta, mientras que el acero transversal se modeló como uniformemente distribuido.

Para el comportamiento mecánico del concreto se tomó un modelo de plasticidad incluido en el código DIANA, que combina los criterios de falla de Von Mises para falla en compresión, y de Rankine para falla en tensión, lo que resulta en la función de falla siguiente (TNO 1996)

$$
\begin{cases}f_{R}\left(\sigma, \kappa_{R}\right)=\sigma_{1}-f_{t}\left(\kappa_{R}\right) & ; \dot{\kappa}_{R}=\dot{\varepsilon}_{1}^{P} \\ f_{V M}\left(\sigma, \kappa_{V M}\right)=\sqrt{\sigma_{1}^{2}-\sigma_{1} \sigma_{2}+\sigma_{2}^{2}}-f_{c}\left(\kappa_{V M}\right) ; \dot{\kappa}_{V M}=\sqrt{\frac{2}{3}\left(\left(\dot{\varepsilon}_{1}^{P}\right)^{2}+\left(\dot{\varepsilon}_{2}^{P}\right)^{2}+\left(\dot{\varepsilon}_{3}^{P}\right)^{2}\right)}\end{cases}
$$

donde $\sigma_{1}$ y $\sigma_{2}$ son los esfuerzos principales, las $\kappa_{i}$ son variables internas de estado o deformaciones plásticas equivalentes; $f_{t}\left(\kappa_{R}\right)$ y $f_{c}\left(\kappa_{V M}\right)$ son las resistencias en tensión y en compresión uniaxiales respectivamente; las $\varepsilon_{i}^{P}$ son las deformaciones plásticas principales; y el 
punto sobre una variable indica derivación con respecto al tiempo. En la fig 3.a se presenta gráficamente esta función. En compresión el comportamiento es lineal hasta un tercio de la resistencia, a partir de este punto la relación $\sigma-\varepsilon$ sigue una ley parabólica, con su máximo en $f_{c}$ y con energía de fractura $G_{f c}$ dada $(\mathrm{TNO}, 1996)$. En la fig 3.b se muestra la ley de endurecimiento. En tensión el comportamiento es lineal hasta $f_{t}$, y a partir de aquí el ablandamiento se modela por medio de una ley exponencial con energía de fractura $G_{f t}$ dada. Físicamente $G_{f}$ es la energía necesaria para generar una superficie unitaria de grieta, es común aceptar que esta cantidad es una característica intrínseca de cada material. Numéricamente es el área bajo la curva esfuerzo contra ancho de grieta. En la fig 4 se indica que el área bajo la curva esfuerzo-deformación es la energía relativa de fractura $g_{f}$. La relación entre la energía de fractura $G_{f}$ y la energía relativa de fractura $g_{f}$ es:

$$
\mathrm{G}_{f}=g_{f} h
$$

donde $h$ es el ancho de banda del material, en el caso de elementos finitos, se ha demostrado que este parámetro depende de la geometría del elemento. Para cuadriláteros es común tomar a $h$ como la raíz cuadrada del área del elemento.

La mampostería tiene un comportamiento mecánico ortótropo, que se acentúa en la etapa de comportamiento no lineal, sin embargo, en el presente trabajo se consideró siempre comportamiento isótropo por varias razones. La primera es que actualmente se cuenta con muy poca información experimental acerca del comportamiento ortótropo de la mampostería, y hasta donde llega el conocimiento de los autores, no hay estudio alguno al respecto hecho con mamposterías mexicanas, por lo que sería muy aventurado intentar asignar propiedades ortótropas a un modelo. Además, un comportamiento isótropo debe ser suficiente para captar la respuesta general de la estructura porque las direcciones en las que ocurre la plastificación y el agrietamiento son muy similares en todo el muro y en toda la historia de carga. Otra razón es que los modelos de plasticidad ortótropos incorporados en DIANA se limitan a comportamiento elasto-plástico perfecto el cual está muy alejado del de la mampostería, sobre todo si se quiere modelar su comportamiento no lineal. Otra limitante para considerar un modelo ortótropo es que con los métodos de análisis actuales no se puede combinar con los modelos de fractura, lo que los hace todavía menos aptos para representar el comportamiento de la mampostería. Es deseable que los modelos de plasticidad ortótropa, como el desarrollado por Lourenço (1996), se tornen disponibles en los programas de análisis no lineal de elementos finitos. Esto permitiría una modelación más precisa que incluya todos los posibles modos de falla de la mampostería, por ejemplo, el deslizamiento a lo largo de las juntas horizontales, que no puede modelarse con un modelo isótropo. 
Para el modelo de comportamiento de la mampostería se utilizó el criterio de Von Mises para falla en compresión, y un criterio lineal para tensión, como se indica en la fig 4.a. El modelo de comportamiento en compresión es parabólico, como en el modelo del concreto, y el de tensión está basado en la teoría de la fractura con ablandamiento de Hordijk (TNO, 1996), fig 4.b. En la mecánica de la fractura se reconoce que al iniciarse la formación de una grieta, la rigidez en cortante del material se reduce. Con este fin se introduce el factor de retención de cortante $\beta$, menor que la unidad y mayor que cero, que multiplica al módulo de cortante $G$, en este caso se tomó un valor constante de $\beta=0.001$. Este valor es demasiado pequeño y no tiene una justificación física o experimental; sin embargo, numéricamente ofrece la ventaja de evitar que los esfuerzos principales giraren lo suficiente como para que se inicie la formación de otra grieta, con diferente orientación, en un mismo elemento.

a)

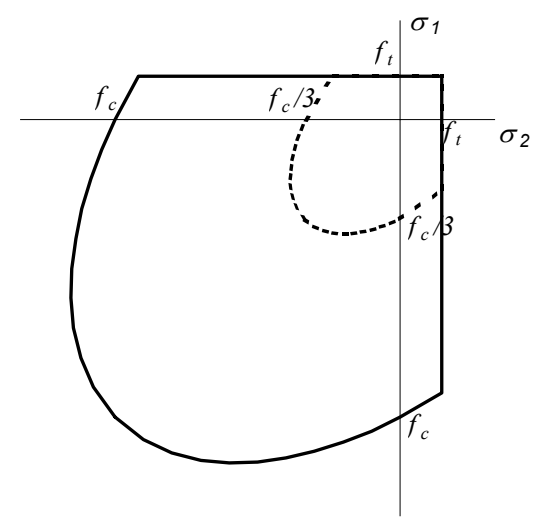

b)

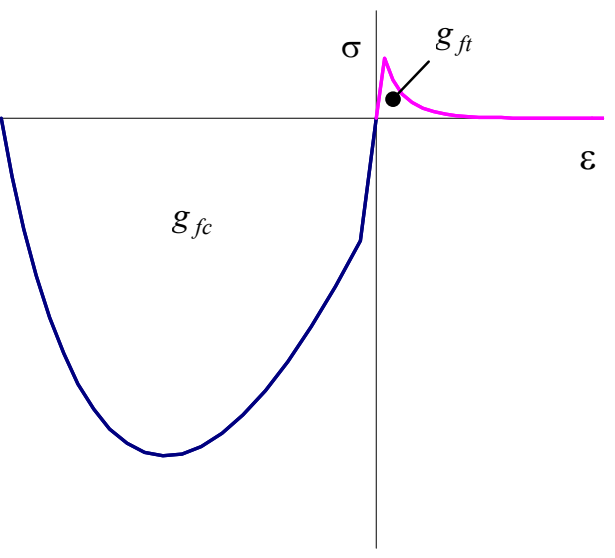

Figura 3. Modelo de comportamiento para el concreto; a) criterio de falla combinado Von MisesRankine; b) ley de endurecimiento

Los elementos interfaz de cuatro nudos que se utilizaron para modelar el contacto entre el muro y el resto de la estructura tienen rigideces elásticas, normal y tangencial, dadas por:

$$
D_{n}=\frac{E_{m}}{t} \quad D_{t}=\frac{G_{m}}{t}
$$

donde $E_{m}$ y $G_{m}$ son los módulos de Young y de cortante del mortero respectivamente, y $t$ es el espesor de la junta que se está representando. Estas rigideces se interpretan físicamente como el valor del esfuerzo que es necesario aplicar a la interfaz para producir un desplazamiento relativo unitario correspondiente con dicho esfuerzo. Para el comportamiento no lineal se utilizó el criterio de falla de Coulomb, con un límite en el esfuerzo de tensión (cut off), como se muestra en 
la fig 5. El comportamiento en dirección normal del elemento es elasto-plástico perfecto. Se utiliza retención de cortante constante, con un valor nulo de la rigidez reducida, lo que implica también comportamiento elasto-plástico perfecto. La matriz de rigideces tangente para este tipo de elementos queda expresada por (TNO 1996)

$$
\mathbf{k}_{\mathbf{t}}=\frac{1}{h+k_{n} \tan (\phi) \tan (\psi)+k_{t}}\left[\begin{array}{cc}
k_{n}\left(h+k_{t}\right) & -k_{n} k_{t} \tan (\psi) \frac{t_{t}}{\left|t_{t}\right|} \\
-k_{n} k_{t} \tan (\phi) \frac{t_{t}}{\left|t_{t}\right|} & k_{t}\left(h+k_{n} \tan (\phi) \tan (\psi)\right)
\end{array}\right]
$$

donde $h=\partial f / \partial \kappa, k_{n}$ y $k_{t}$ son las rigideces elásticas normal y tangencial respectivamente, $\phi$ y $\psi$ son los ángulos de fricción y de expansión respectivamente, y $t_{t}$ es el esfuerzo tangencial. Es evidente de la ec 4 que si el ángulo de fricción es diferente que el de expansión, la matriz resulta ser asimétrica. La expansión depende del nivel de esfuerzos confinantes en razón inversa, del grado de rugosidad de las superficies en contacto, y de la cantidad de desplazamiento tangencial. Inicialmente tiene un valor alto $(0.2<\tan (\psi)<0.75)$ y a medida que se produce el deslizamiento, las rugosidades se liman y la expansión tiende a cero. Lourenço (1996) encuentra que suponer un valor nulo para este parámetro da buenos resultados. El ángulo de fricción también disminuye con el deslizamiento, hasta un valor residual. Lo anterior implica que en general se tienen valores diferentes para $\phi$ y $\psi$, lo que, a su vez, produce matrices tangentes asimétricas.

Para el acero de refuerzo se supone un comportamiento elasto-plástico perfecto, y se considera que existe adherencia perfecta con el concreto. Esta hipótesis es bastante razonable en marcos con muros de relleno, según los resultados que reportan Mehrabi et al (1994), quienes
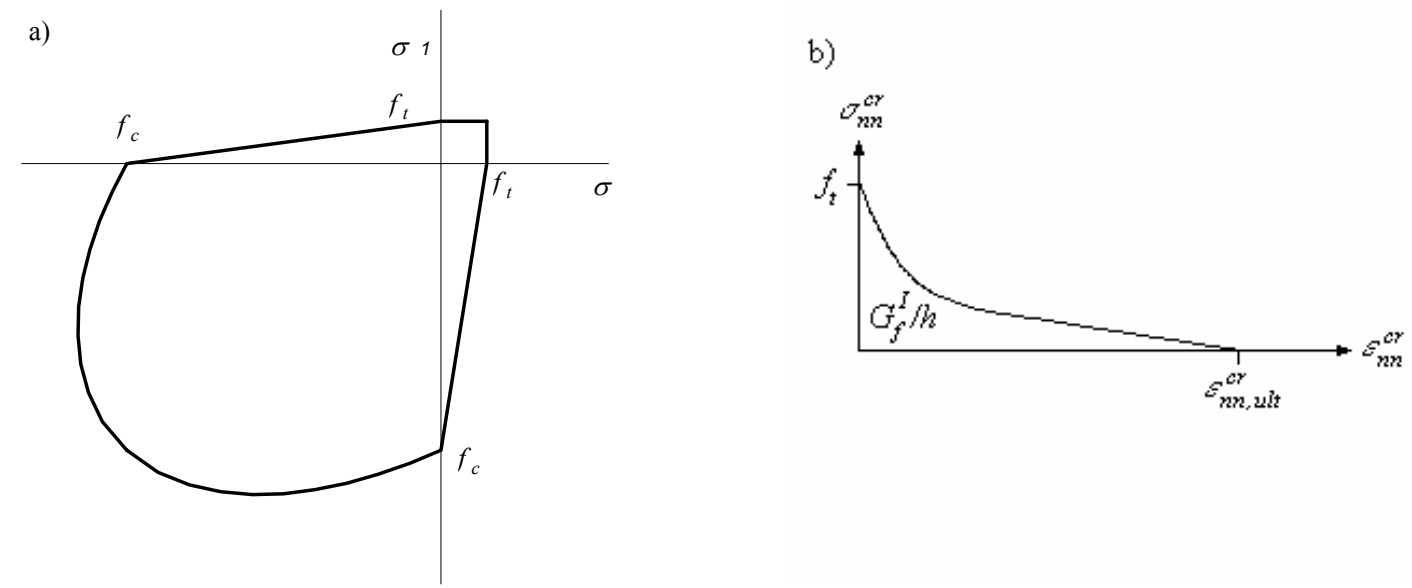

Figura 4. Modelo de fractura para mampostería; a) criterio de falla; b) modelo de ablandamiento de Hordijk (TNO 1996). 
analizan un marco de concreto reforzado con y sin relleno, y con y sin deslizamiento del acero de refuerzo. Encuentran que en el caso del marco sin relleno, el deslizamiento del refuerzo influye significativamente en la respuesta, mientras que en el marco con relleno este fenómeno no es significativo.

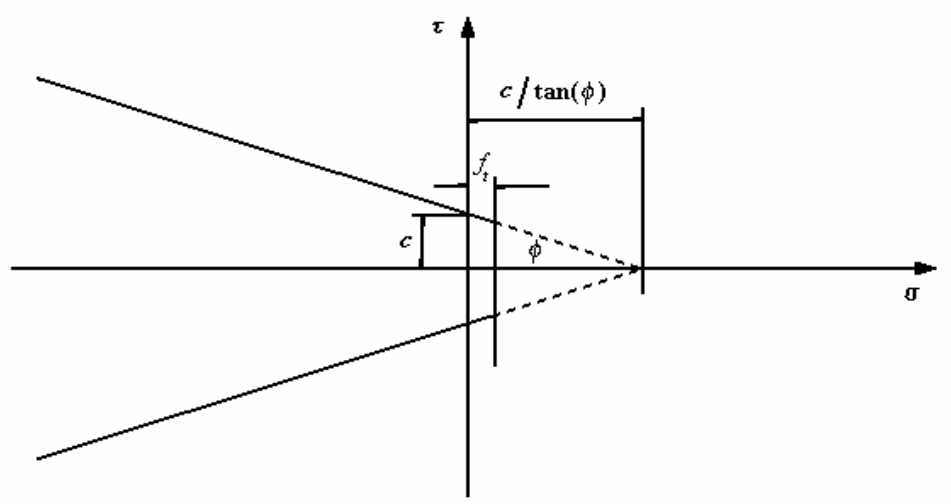

Figura 5. Criterio de falla de Coulomb.

Para encontrar la solución de sistemas no lineales se recurre a procedimientos aproximados que combinan métodos incrementales y métodos iterativos. Para este modelo se intentaron varios de los métodos iterativos más comunes y considerados los más eficientes, como son: Newton-Raphson, Newton-Raphson modificado, el de la matriz secante, entre otros; sin embargo, no se pudo convergir a soluciones más allá de unos cuantos incrementos de carga, ya sea porque no se alcanzaba el criterio de convergencia, o porque el error se hacía mayor con cada iteración, aún usando técnicas avanzadas como el control de la longitud de arco, o el tamaño óptimo del incremento de carga ("line search"). El método que permitió encontrar soluciones aproximadas para cualquier nivel de deformación fue el de la matriz de rigidez lineal, que consiste en utilizar durante todo el proceso la matriz de rigideces que se obtiene inicialmente, antes de que ocurra ningún comportamiento no lineal. Este método tiene las ventajas de que la matriz de rigideces es positiva definida y simétrica, y que se factoriza una sola vez en todo el proceso. Sin embargo, tiene las desventajas de requerir un mayor número de iteraciones para alcanzar la convergencia, comparado con otros métodos, y que la solución aproximada oscila mucho alrededor de la solución exacta real (fig 7). Cabe mencionar que este método iterativo puede conducir a que el análisis siga rutas de carga-deformación erróneas; sin embargo, los resultados obtenidos en este caso parecen ser correctos, puesto que las configuraciones deformadas, los patrones de agrietamiento, y las curvas fuerza-desplazamiento concuerdan con el comportamiento esperado. 


\section{EJEMPLO DE APLICACIÓN}

Con el fin de calibrar el modelo numérico y obtener mayor información del comportamiento de marcos con muros de relleno, se elaboró un modelo de elementos finitos con base en los datos reportados por Meli y Salgado (1969) de uno de sus ensayes experimentales. Aunque estrictamente el modelo experimental es de mampostería confinada, el comportamiento de este tipo de estructuras es muy similar al de los marcos con muros de relleno, además, no se contaba con información experimental detallada de algún experimento en un marco relleno auténtico. Las características geométricas del modelo se muestran en la fig 6.a. Las piezas eran tabiques de barro recocido, con dimensiones nominales de $6 \times 12 \times 24 \mathrm{~cm}$, y con un módulo de Young de $E_{p}=11,000 \mathrm{~kg} / \mathrm{cm}^{2}$. El mortero empleado en las juntas tenía una proporción de 1:3 en cemento:arena con una resistencia a la compresión $f_{b}=285 \mathrm{~kg} / \mathrm{cm}^{2}$. La resistencia a la compresión de la mampostería era de $f_{m}=102 \mathrm{~kg} / \mathrm{cm}^{2}$. La resistencia a la compresión del concreto del marco era $f_{c}^{\prime}=237 \mathrm{~kg} / \mathrm{cm}^{2}$. Los castillos estaban reforzados con cuatro varillas longitudinales de $5 / 8$ in de diámetro, y estribos de $1 / 4$ in a cada $20 \mathrm{~cm}$. El acero de refuerzo era de tipo estructural con esfuerzo de fluencia nominal de $4,000 \mathrm{~kg} / \mathrm{cm}^{2}$. Los castillos se colaron una vez terminada la construcción del muro. La carga horizontal se aplicaba progresivamente para producir incrementos de deformación angular prefijados, controlados por medio de la deformación de una de las diagonales.

Meli y Salgado (1969) reportan que en el experimento el muro se agrietó cuando la carga lateral alcanzó las 6.43 ton, y la deformación angular era de $1.11 \times 10^{-3}$. Después la carga se incrementó hasta un máximo de 11.00 ton cuando el muro había alcanzado una deformación angular de $13.4 \times 10^{-3}$.

La malla de elementos finitos empleada se muestra en la fig 6.b. Los elementos interfaz que unen al muro con el resto de la estructura tienen un espesor nulo. Después de un proceso de calibración en el que se mantuvieron constantes las propiedades del marco de concreto reforzado y de la interfaz, se llegó a los parámetros que se indican en la tabla 1, el espesor de la junta entre muro y marco se supuso de $1 \mathrm{~cm}$. Una descripción detallada del proceso de calibración puede consultarse en Orduña (2000). Respecto a los valores asentados en la tabla 1 se pueden hacer los siguientes comentarios. El esfuerzo de fluencia del acero en tensión es el reportado por Meli y Salgado (1969), para el esfuerzo de fluencia en compresión se tomó el mismo valor, y para el módulo de Young, un valor comúnmente aceptado en la literatura. La resistencia en compresión del concreto es la obtenida experimentalmente; mientras que para la resistencia en tensión se tomó el 10\% del valor anterior; el módulo de Young se calculó de acuerdo con DDF (1996); el módulo de Poisson es el comúnmente aceptado en la literatura. Los valores de las energías de fractura en compresión y en tensión, difícilmente pueden obtenerse de manera experimental, los 
a)
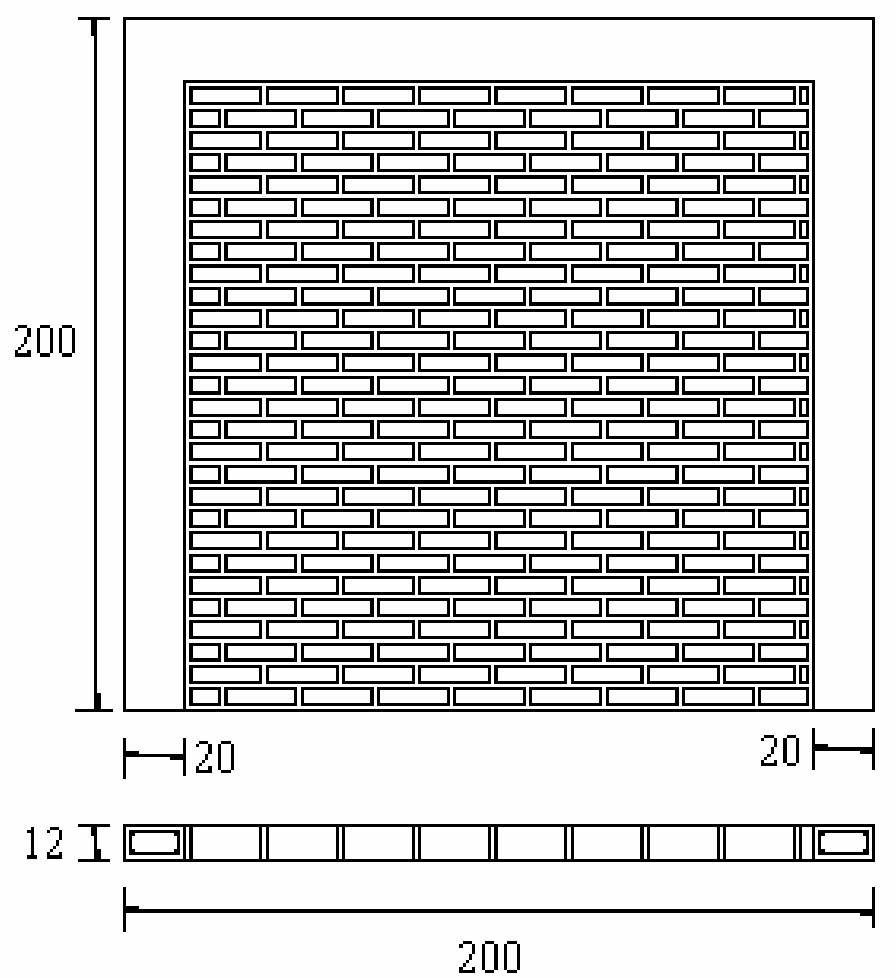

b)

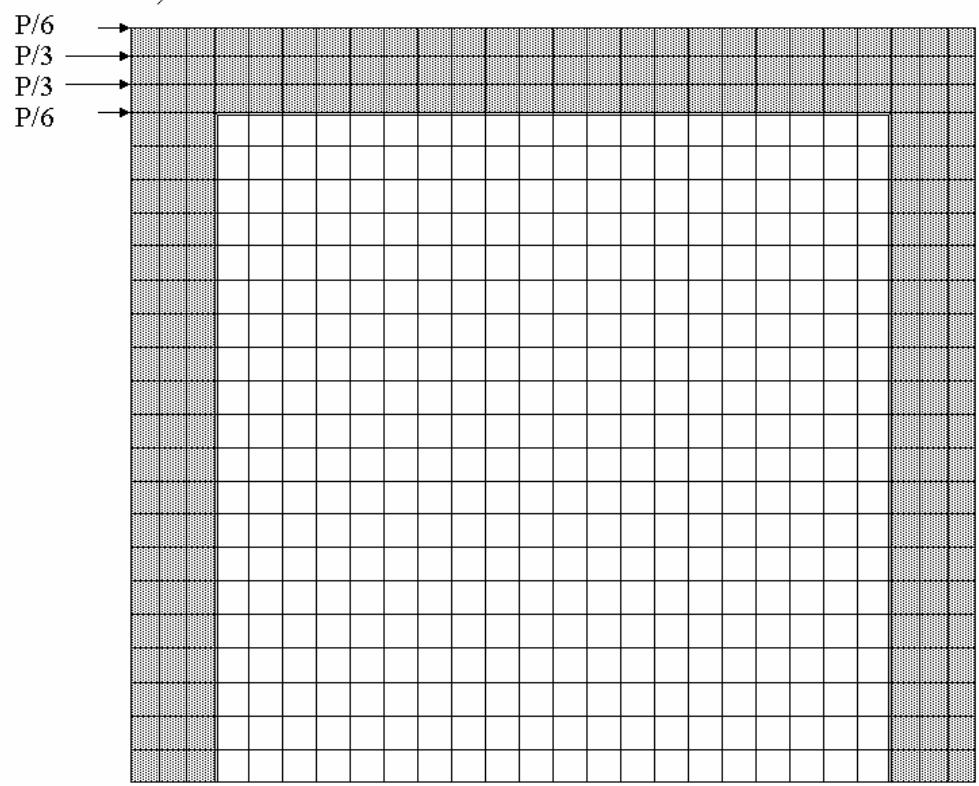

Figura 6. Modelo de marco y muro confinado; a) características geométricas del modelo experimental de Meli y Salgado (1969); b) malla de elementos finitos del modelo numérico. 
Tabla 1. Parámetros del modelo numérico.

\begin{tabular}{|c|c|c|c|c|c|c|c|c|c|}
\hline & $\begin{array}{c}E \\
\mathrm{~kg} / \mathrm{cm}^{2}\end{array}$ & $\begin{array}{l}v \\
-\end{array}$ & $\begin{array}{c}f_{c} \\
\mathrm{~kg} / \mathrm{cm}^{2}\end{array}$ & $\begin{array}{c}G_{f_{c}} \\
\mathrm{~kg} . \mathrm{cm} / \mathrm{c} \\
\mathrm{m}^{2}\end{array}$ & $\begin{array}{c}f_{t} \\
\mathrm{~kg} / \mathrm{cm}^{2}\end{array}$ & $\begin{array}{c}G_{f t} \\
\mathrm{~kg} \cdot \mathrm{cm} / \mathrm{c} \\
\mathrm{m}^{2}\end{array}$ & $\begin{array}{c}\mathrm{c} \\
\mathrm{kg} / \mathrm{cm}^{2}\end{array}$ & $\begin{array}{c}\tan (\phi) \\
-\end{array}$ & $\begin{array}{c}\tan (\psi) \\
-\end{array}$ \\
\hline Mampostería & 8000 & 0.25 & 14.0 & 2.0 & 2.2 & 0.01 & - & - & - \\
\hline Concreto & $\begin{array}{c}1.2 \times 10 \\
5\end{array}$ & 0.20 & 237 & 5.0 & 23.7 & 0.05 & - & - & - \\
\hline Acero & $2 \times 10^{6}$ & - & 4000 & - & 4000 & - & - & - & - \\
\hline Interfaz & 8000 & 0.25 & - & - & 0.0 & - & 2.5 & 0.75 & 0.0 \\
\hline
\end{tabular}

valores usados han dado buenos resultados en análisis numéricos de otros autores, por ejemplo: Lourenço (1996) o Rots (1988). Para la mampostería se tomó un módulo de Poisson comúnmente aceptado en la literatura. Las resistencias y las energías de fractura en compresión y tensión, así como el módulo de Young de la mampostería, fueron los parámetros que más sufrieron ajustes en el proceso de calibración, los valores indicados son los que dieron mejores resultados en cuanto a que la respuesta del modelo numérico fuera similar a la del experimental. De hecho puede observarse que la resistencia en compresión en el modelo numérico es muy inferior a la medida experimentalmente en prismas de mampostería. Este hecho es indicativo de que el modelo numérico aún tiene muchas deficiencias para reproducir correctamente el comportamiento de este tipo de estructuras, pero también de que es necesario obtener información experimental acorde con las necesidades de los modelos numéricos actuales. Finalmente, para la interfaz entre muro y marco, se consideró una cohesión de $c=2.5 \mathrm{~kg} / \mathrm{cm}^{2}$ que es un valor recomendado por Meli (1979) basado en evidencia experimental; y la tangente del ángulo de fricción se tomó igual a 0.75, este es un valor razonable según varios autores (Lourenço 1996, Mehrabi et al.1996, Meli 1979). El ángulo de expansión se tomó igual a cero porque este valor ha dado buenos resultados en otros trabajos (Lourenço 1996).

En la fig 7 se comparan las curvas carga contra deformación angular de los modelos experimental y numérico. En esta figura se observa un comportamiento muy similar de los dos modelos hasta antes del agrietamiento diagonal del muro, en donde el modelo experimental reduce ligeramente su carga antes de retomar una rigidez positiva. En el modelo numérico el agrietamiento ocurre a una carga ligeramente menor que en el experimental, sin embargo, después del agrietamiento, el comportamiento vuelve a ser muy similar en ambos. En la última etapa, el modelo experimental pierde rigidez más rápidamente que el numérico. Para explicar este comportamiento se han considerado dos hipótesis aún no verificadas. La primera es que el comportamiento de estructuras en las que el agrietamiento ocurre como una sola grieta, no puede ser modelado adecuadamente por medio del enfoque de la fractura distribuida, en cuyo caso la 
fractura discreta debería de dar mejores resultados, aunque a un costo mucho mayor. La otra posible explicación es que en los modelos de comportamiento considerado no se reconoce ninguna reducción en la resistencia en compresión de los materiales cuando se ha iniciado el agrietamiento.

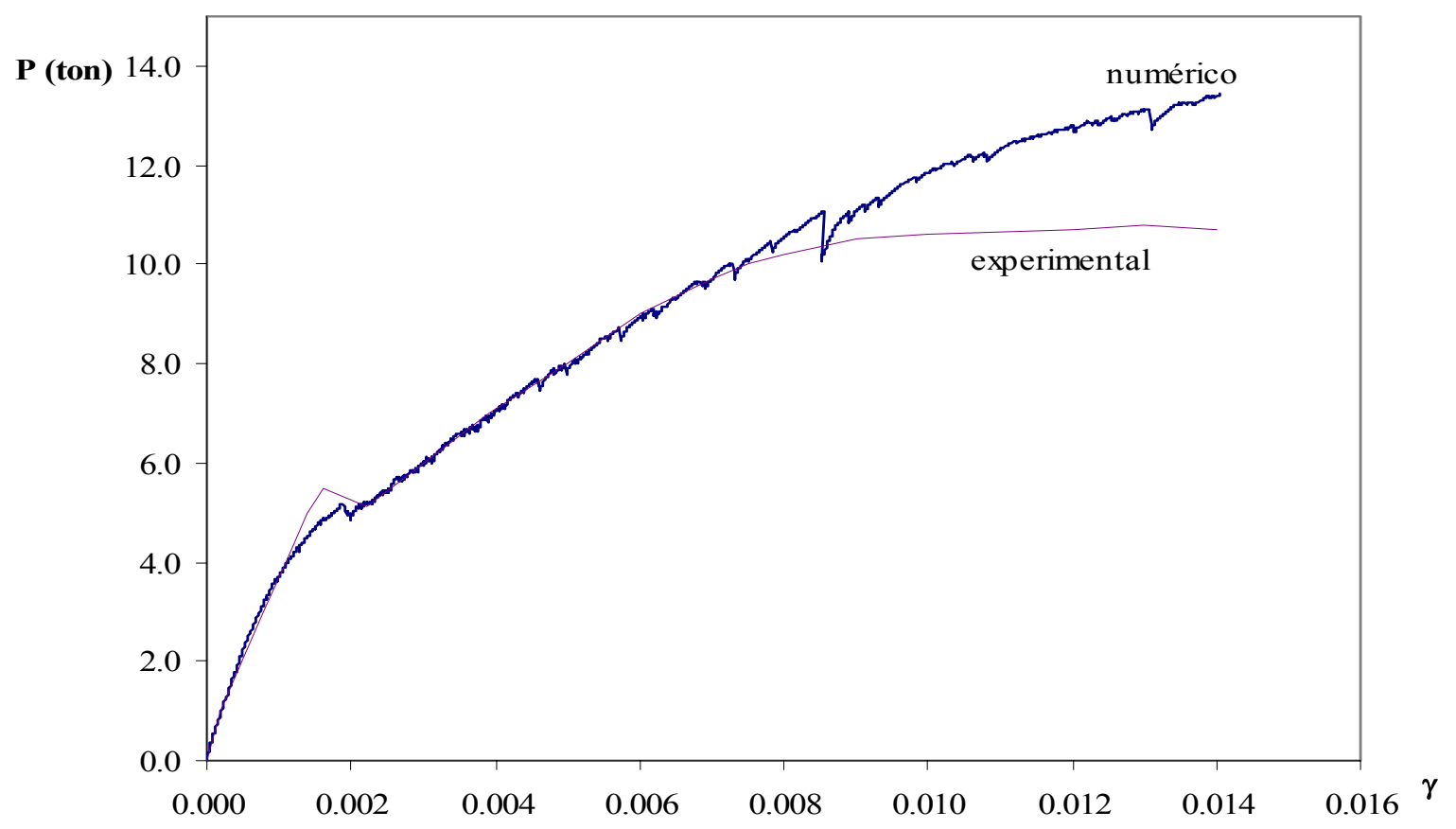

Figura 7. Gráfica carga contra deformación angular

\section{PROPUESTA DE MODELO DE DIAGONALES}

Como una aplicación práctica de los resultados obtenidos de modelos numéricos como el presentado en la sección anterior, se propone el desarrollo de un modelo simplificado de muro para el análisis de marcos con rellenos. El objetivo principal de proponer un nuevo modelo para el análisis de muros diafragma es intentar reproducir lo mejor posible la distribución de elementos mecánicos en el marco obtenidos mediante un análisis no lineal, con el fin de que el diseño del mismo sea congruente con las solicitaciones a las que estará expuesto durante un sismo intenso; al mismo tiempo que se pueda reproducir adecuadamente el comportamiento global. Debido a que únicamente se cuenta con resultados de un modelo de elementos finitos, no fue posible calibrar el modelo simplificado para su aplicación general, por ello, la propuesta se limita a sentar las bases para el desarrollo de dicho modelo. Se ilustra, mediante el ejemplo del muro estudiado en la sección anterior, que es posible definir los parámetros del modelo simplificado de tal manera que se cumpla con el objetivo señalado arriba. 
Los elementos mecánicos cambian no sólo en magnitud, sino también en distribución, debido principalmente al cambio en la longitud de contacto con el muro, a medida que ocurre el daño en la estructura. Tratar de modelar los cambios en la longitud de contacto implicaría que no sólo las propiedades mecánicas del modelo fueran cambiantes, sino también su geometría, y esto lo haría demasiado complejo para su uso en la práctica común de la ingeniería. Por lo anterior se consideró que el modelo tuviera una longitud de contacto constante para todo su comportamiento. La longitud de contacto que se consideró más representativa es la que se presenta inmediatamente después de que ocurre el agrietamiento del muro. Por una parte, porque la carga que transmite el muro no aumenta mucho después del agrietamiento; y por otra parte, porque se espera que en una estructura bien diseñada, para niveles de desempeño relacionados con el daño, la mayoría de los muros deben alcanzar al menos la carga de agrietamiento durante el sismo más intenso que probablemente se presente en su vida útil.

El modelo que se propone consiste en sustituir al muro por tres elementos armadura que únicamente pueden tomar fuerzas axiales de compresión (fig 8), de manera similar al propuesto por Chrysostomou et al (1992). El elemento en la diagonal modela el comportamiento de una

a)

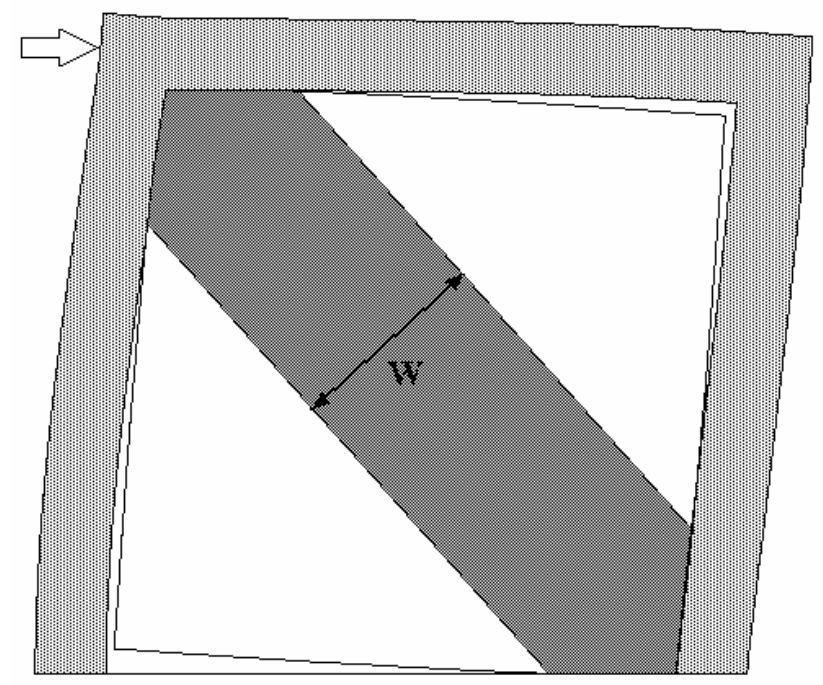

b)

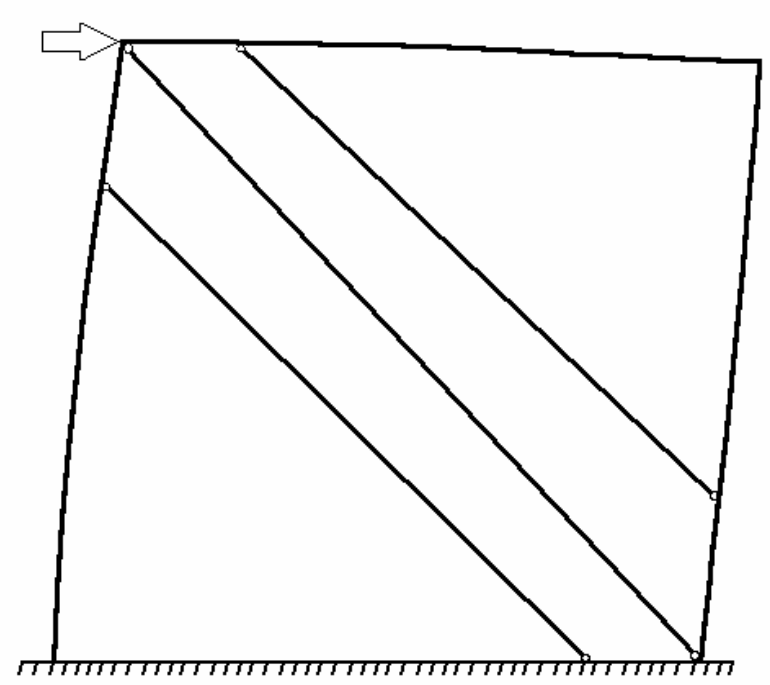

Figura 8. Modelo de tres diagonales; a) Idealización del trabajo de un muro como un puntal en compresión; b) modelo propuesto para análisis.

franja de muro que inicialmente toma buena parte de la carga, pero cuya contribución en la transmisión de cargas disminuye notablemente cuando ocurre el agrietamiento del muro. Los 
elementos excéntricos inicialmente transmiten una parte pequeña de la carga, pero modifican las distribuciones de elementos mecánicos del marco, y permiten, además, tomar en cuenta la posible excentricidad con la que se transmite la carga del muro al marco. Cuando ocurre el agrietamiento del muro y el elemento central pierde rigidez, los elementos excéntricos toman un porcentaje mayor de la carga. Esto es congruente con lo observado en el modelo de elementos finitos, en donde, para las etapas finales, se observa una reducción de los esfuerzos principales en la franja central del muro, y la formación de franjas laterales a través de las cuales se transmiten los esfuerzos.

A los elementos se les asigna comportamiento elasto-plástico perfecto, porque es el más sencillo para representar comportamiento no lineal. La rigidez inicial del elemento central se define a partir del estado de la estructura antes de producirse el agrietamiento del muro. Cuando se alcanza este estado, la diagonal central fluye. El segundo estado que se considera importante es el correspondiente a la deformación angular de $\gamma=0.006$. Este valor es en cierto modo arbitrario, deben hacerse estudios encaminados a determinar este parámetro, e incluso este segundo estado o estado último puede considerarse dependiente del nivel y tipo de desempeño que se quiera lograr. Las rigideces de las diagonales excéntricas están definidas por el comportamiento entre el agrietamiento del muro y la deformación máxima; estos elementos fluyen cuando se alcanza este estado.

Con el fin de verificar que es posible definir los parámetros de un modelo como el propuesto, de manera tal que se cumplan los objetivos señalados, se procedió a calibrar un modelo con la estructura estudiada en el capítulo anterior. Debido a que lo que se pretende validar es el modelo que sustituye al muro, el modelo del marco se conservó exactamente igual al descrito en la sección anterior; se eliminaron los elementos que representan al muro y a la interfaz, y se agregaron los tres elementos barra del modelo propuesto. Existen tres conjuntos de variables que definen el comportamiento del modelo propuesto, el primero es la ubicación de las diagonales excéntricas, el segundo está integrado por las rigideces de los elementos, y el tercero por sus resistencias.

La ubicación de las diagonales debe estar relacionado con la longitud de contacto entre el muro y cada uno de los elementos que lo confinan (en este caso se incluye la viga de cimentación). Se observó que una buena elección es colocar los extremos de los elementos diagonales en los puntos donde se inicia el contacto al momento de producirse el agrietamiento del muro; sin embargo, es necesario considerar un mayor número de casos para dar una recomendación general en este sentido. 
Para definir las rigideces y resistencias de cada uno de los elementos diagonales, se estudió la distribución de los esfuerzos de contacto entre muro y marco en los estados de agrietamiento y deformación máxima. Se supuso que la diagonal central debería tomar la fuerza resultante de los esfuerzos de contacto en zonas definidas, tanto de la viga como de la columna, con base en la recomendación que se hace en DDF (1996), respecto a que el cortante con el que debe diseñarse un elemento de concreto reforzado es aquel que se presenta a un peralte del paño del apoyo. De esta forma las dos zonas de contacto entre muro y marco quedan divididas en tres subzonas, y la resultante de los esfuerzos de contacto en ellas se pueden asociar con cada una de las tres diagonales del modelo. Estas subzonas de contacto definen, asimismo, las áreas transversales de los elementos diagonales. Nuevamente es importante aclarar que el criterio adoptado para dividir la zona de contacto en tres subzonas es arbitrario, cuando se cuente con un mayor número de casos estudiados, se podrá determinar un criterio más adecuado.

La fig 9 muestra la proporción de la carga lateral que toman el marco y el muro para algunos pasos de carga, desafortunadamente no se cuenta con un desglose de cargas similar para el modelo experimental, por lo que no se puede establecer una comparación. Para separar la carga que toma el marco se sumaron los cortantes en los extremos inferior de la columna izquierda y superior de la columna derecha. Se hizo de este modo porque el cortante en el extremo inferior de la columna derecha incluye parte de la carga que toma el muro y que transmite nuevamente al marco a través de esta columna. El cortante que toma el muro se calculó como la diferencia entre el total y el que toma el marco. Se puede observar que el cortante en el muro se incrementa con una tendencia bilineal, esto refuerza la propuesta de hacer que la diagonal central fluya al producirse el agrietamiento del muro. De esta forma la suma de las rigideces iniciales de las tres diagonales debe ser la rigidez de la primera rama de esta curva, mientras que la segunda rama, define la suma de las rigideces de las diagonales excéntricas.

En la fig 10 se muestra el modelo con las tres diagonales; al lado de cada una de ellas aparece un número para identificarlas. Las diagonales excéntricas se conectan en los puntos en los que terminan las zonas de contacto. Puede observarse que los extremos de estas diagonales no coinciden con nudos del modelo del marco; esto se hizo así porque en el último elemento interfaz donde hay contacto, existe esfuerzo en un extremo, mientras que en el otro extremo el esfuerzo es nulo, por lo que se considera que deja de haber contacto a la mitad del elemento. Para hacer la conexión se restringieron linealmente los desplazamientos de los nudos extremos de la diagonales, en función de los desplazamientos de los nudos adyacentes del modelo del marco. 


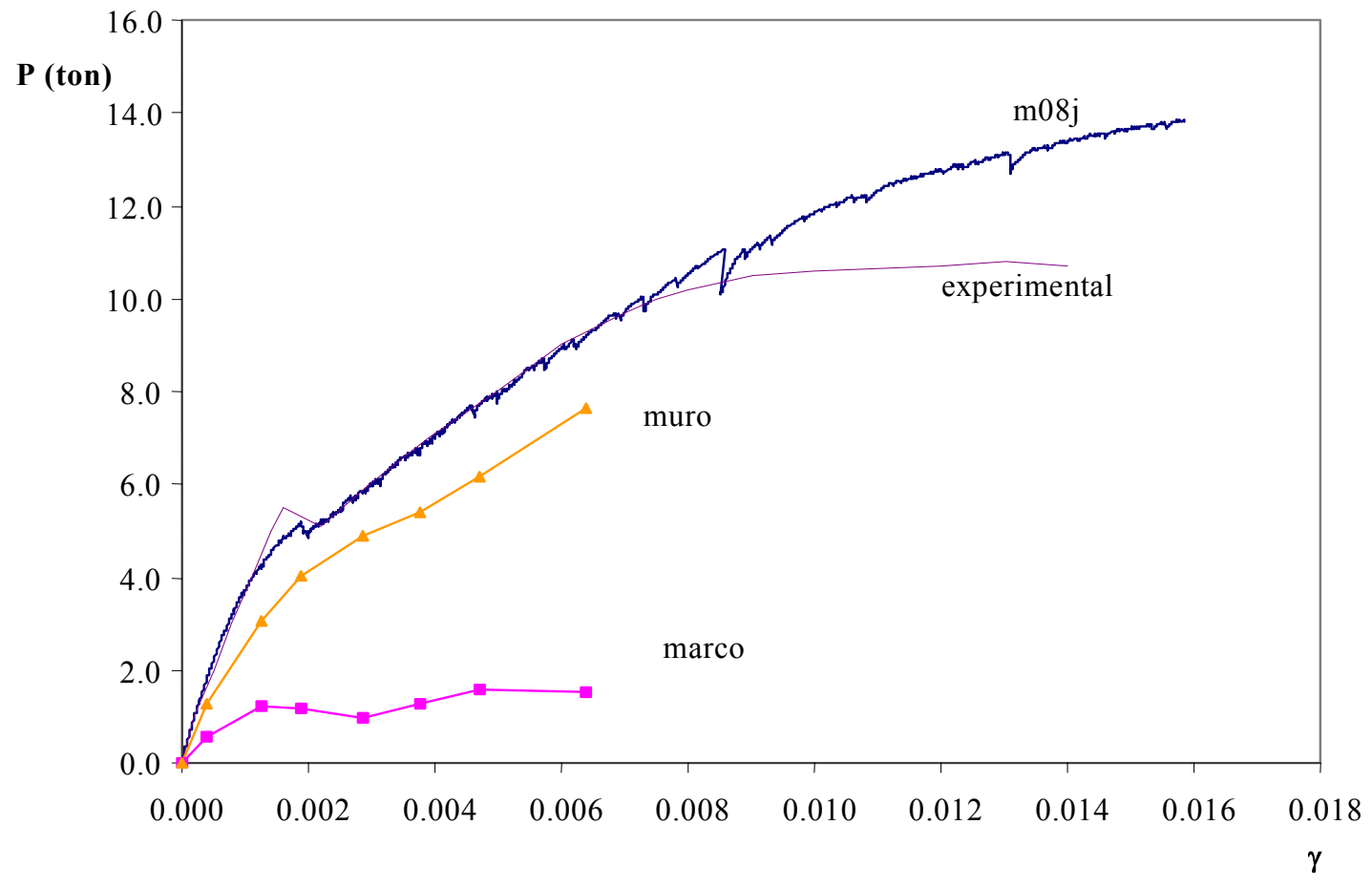

Figura 9. Cortantes que toman el muro y el marco.

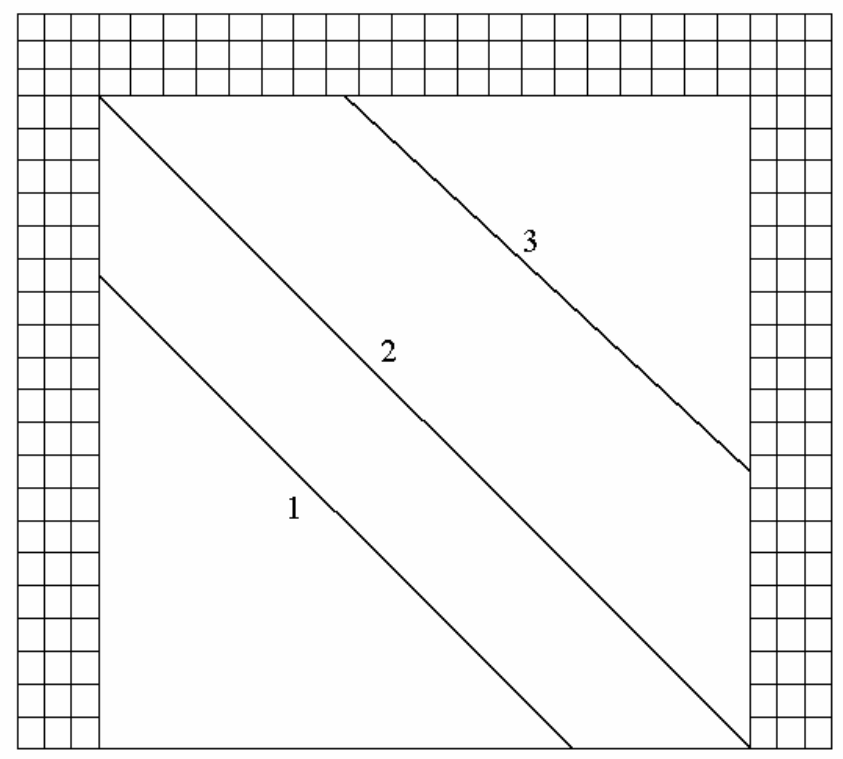

Figura 10. Modelo de tres diagonales y numeración de las mismas. 
Se hicieron una serie de análisis de este modelo, en los que se fueron modificando los módulos de Young de las diagonales hasta obtener un comportamiento global similar al presentado por los modelos de elementos finitos y experimental. Las resistencias se definieron de manera que la diagonal central fluye cuando alcanza la carga de agrietamiento, mientras que las diagonales externas fluyen cuando alcanzan la carga correspondiente con el estado último. Las propiedades de los elementos equivalentes se determinaron por prueba y error; sin embargo, la intención es que, una vez que se cuente con una cantidad suficiente de modelos así obtenidos para diferentes casos, se puedan obtener reglas de aplicación general.

De la forma descrita se llegaron a definir los valores de los parámetros de los elementos diagonales que se indican en la tabla 2 con los cuales se obtiene la curva carga contra deformación angular que se muestra en la fig 11. En esta figura se incluyen, con fines comparativos, las curvas obtenidas con el modelo de elementos finitos y con el modelo experimental, además, se muestran los cortantes que toman el muro en el modelo de elementos finitos, y las diagonales en el modelo simplificado. Se observa que el modelo de tres diagonales se comporta en forma muy similar a los otros dos. La fluencia de las diagonales excéntricas ocurre antes de la deformación angular de 0.006; sin embargo, esto hace que las curvas sean más parecidas entre sí. Asimismo, se observa que el cortante que toman las diagonales en el modelo simplificado concuerda aproximadamente con el que toma el muro en el modelo de elementos finitos hasta la deformación de 0.006. Poco antes de este punto, las diagonales han fluido y no tienen más rigidez, mientras que el muro sigue tomando más carga aparentemente. Ya se comentó en el capítulo anterior que esta falta de ablandamiento del muro es la posible causa de las discrepancias para deformaciones grandes entre los modelos experimental y numérico.

Tabla 2. Propiedades de los elementos diagonales equivalentes.

\begin{tabular}{|c|c|c|c|}
\hline Elemento & $A$ & $E$ & $f_{m}$ \\
\hline & $\mathrm{cm}^{2}$ & $\mathrm{~kg} / \mathrm{cm}^{2}$ & $\mathrm{~kg} / \mathrm{cm}^{2}$ \\
\hline 1 & 270 & 6,000 & 12.0 \\
\hline 2 & 340 & 11,000 & 8.0 \\
\hline 3 & 340 & 6,000 & 12.0 \\
\hline
\end{tabular}




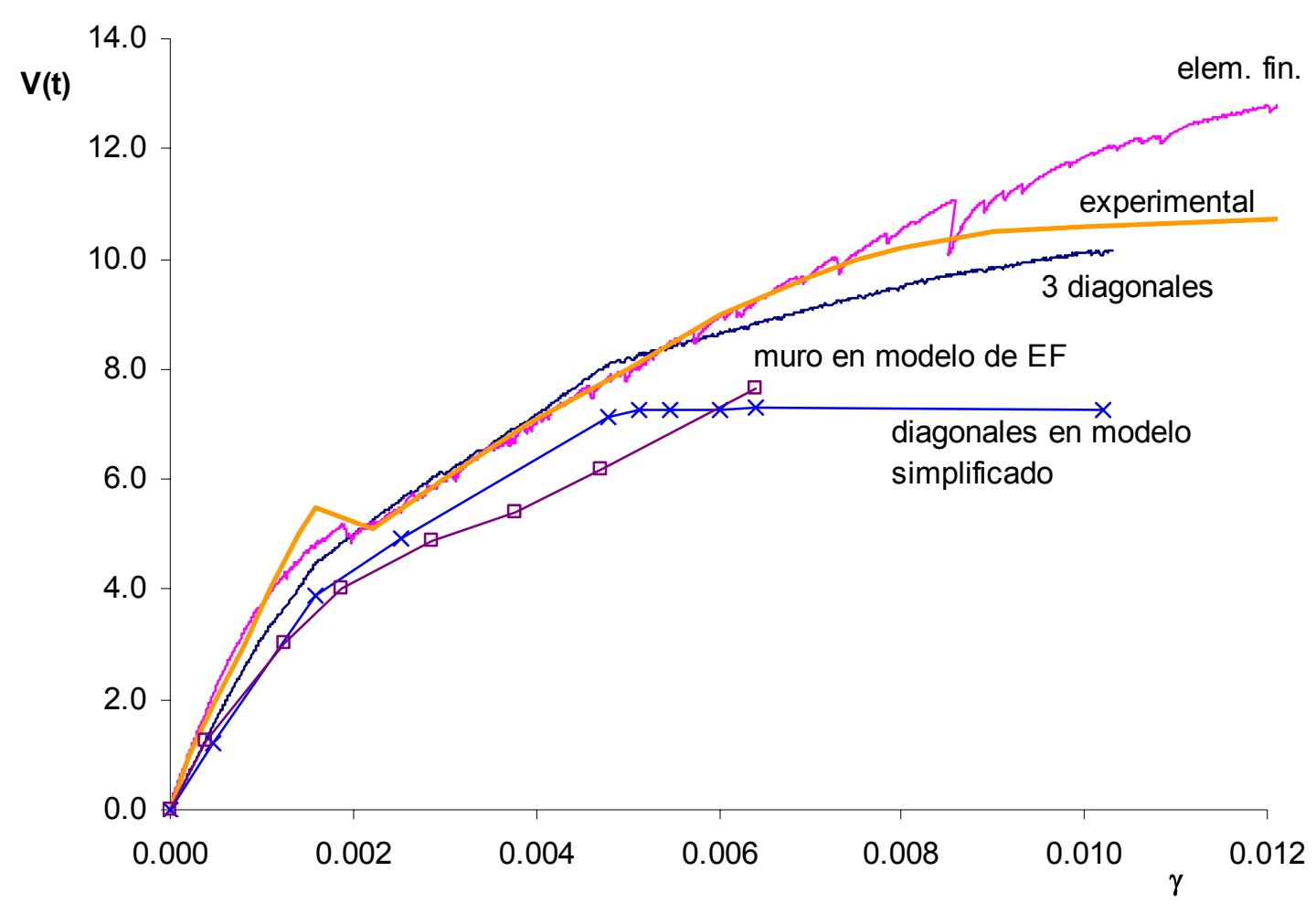

Figura 11. Gráficas carga contra deformación angular de los tres modelos, del muro en el modelo de elemento finitos y de las diagonales en el modelo simplificado.

En la fig 12 se muestran los elementos mecánicos obtenidos con ambos modelos en el estado de deformación máxima. Las tres gráficas superiores muestran los diagramas de momento flexionante. La de la izquierda corresponde con la columna izquierda del marco, el eje vertical es el eje del elemento, mientras en que el eje horizontal se representan los valores del momento. La gráfica de la derecha corresponde con la columna derecha y los ejes tienen significados análogos a los de la columna opuesta. La gráfica central representa el momento flexionante sobre la viga en su eje vertical, mientras que el eje horizontal corresponde con el eje del elemento estructural. Las gráficas de la parte central e inferior de la figura representan las fuerzas cortante y normal, respectivamente, y su interpretación es análoga a las gráficas de momento flexionante. Se aprecia que existe buena semejanza entre ambos modelos. Las diferencias en las formas de los diagramas eran de esperarse por la distinta manera en que se transmiten las cargas al marco. Sin embargo, el modelo de tres diagonales, a diferencia de los basados en una sola diagonal, permite aproximar la variación de los elementos mecánicos en los extremos de los elementos del marco. Aquí es importante mencionar que, para un mayor refinamiento del modelo propuesto, se debería estudiar la posible modificación de las rigideces de las porciones de los elementos del marco que se encuentran entre la diagonal y el nudo. Esto con el fin de igualar las deformaciones en estos tramos, que en el caso real ocurren por fuerzas distribuidas, mientras que en el modelo 

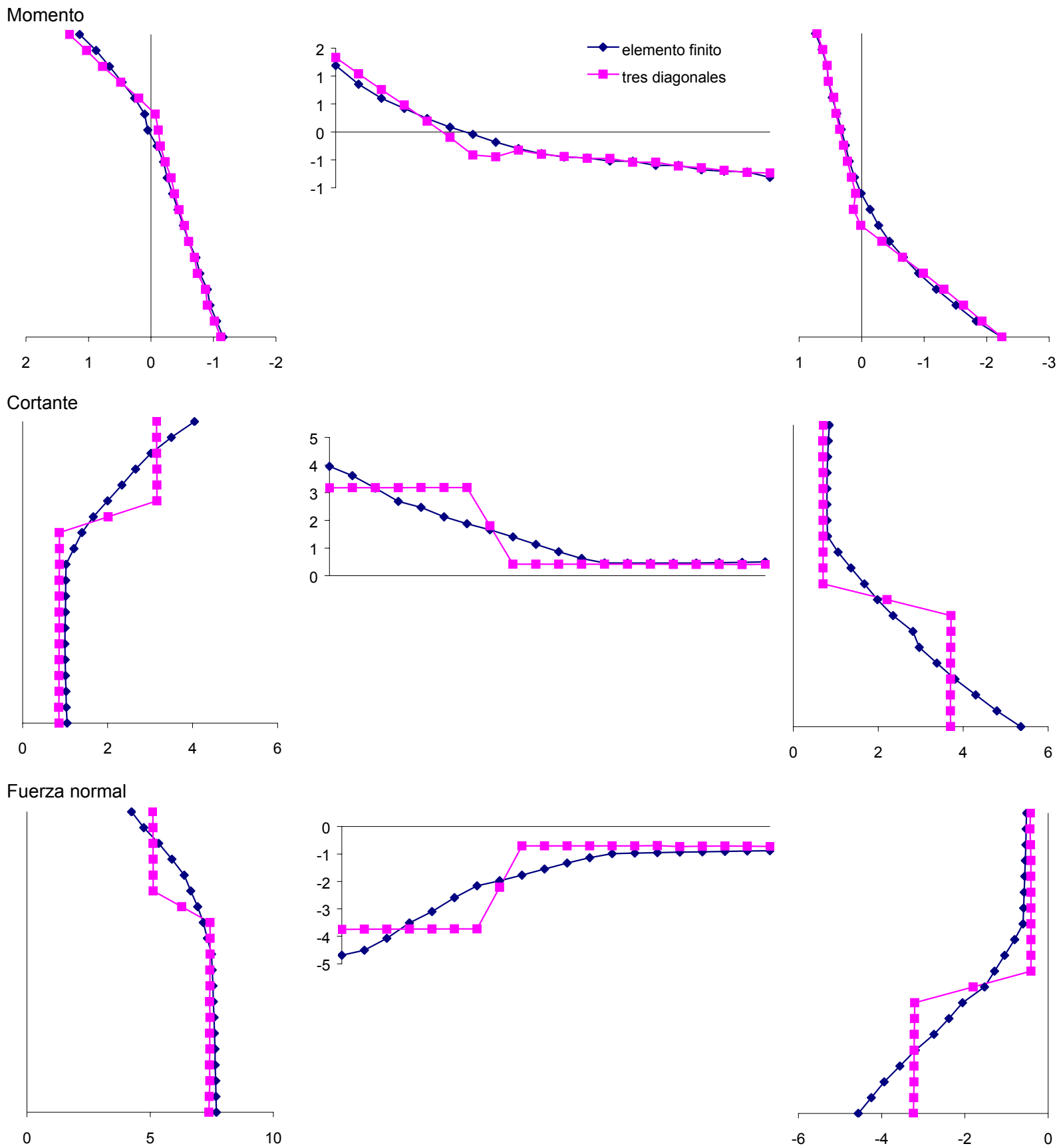

Figura 12. Comparación de elementos mecánicos en el marco entre el modelo simplificado y el modelo de elementos finitos para la deformación máxima. 
simplificado ocurren por fuerzas concentradas con cambios bruscos en las fuerzas cortante y normal, y en la pendiente del diagrama de momentos. Además, en el caso real, se transmiten esfuerzos tangenciales en el paño de los elementos del marco, lo cual va en contra de una de las hipótesis básicas de la teoría de vigas. Esto justifica realizar un estudio detallado del comportamiento de los elementos del marco en la zona de contacto con el muro, con fines no únicamente de análisis, sino para estudiar las implicaciones que este fenómeno pudiera tener en el diseño.

Las propuestas actuales de diseño sísmico basado en el desempeño de las estructuras, parten del supuesto de que el comportamiento de una estructura se puede caracterizar suficientemente, si el modelo de la estructura se somete a una carga lateral monótonamente creciente, y se obtiene la curva carga contra desplazamiento lateral (evidentemente que los escalares carga y desplazamiento laterales deben ser definidos de manera conveniente). Esta curva toma en cuenta el comportamiento no lineal de la estructura, y se interpreta como la envolvente del comportamiento de la estructura cuando es sometida a cargas cíclicas alternantes. Debe notarse que el modelo propuesto depende de la dirección de la carga, para carga en la dirección contraria, debe cambiarse la posición de los elementos barra, orientándolos con la otra diagonal del muro. Los efectos de la degradación de resistencia y de rigidez, disipación de energía y daño progresivo por comportamiento histerético, entre otros; son tomados en cuenta por este enfoque mediante la definición de espectros de diseño adecuados para cada tipo de estructura, material estructural o tipo de desempeño que se quiere controlar. Se deben hacer estudios adicionales para definir tales espectros, estudios que deberán tomar en cuenta los efectos mencionados arriba, pero que salen de los alcances del presente trabajo.

Por otro lado, es importante mencionar que el presente trabajo se limita a proponer las bases para el desarrollo de un modelo simplificado de análisis. Para desarrollar completamente el modelo simplificado, primero es necesario contar con técnicas de modelado por elementos finitos más adecuadas a este tipo de estructuras, calibradas y comprobadas con un número suficiente de ensayos experimentales, para, como segundo paso, realizar estudios paramétricos que permitan establecer reglas generales para definir los parámetros del modelo simplificado en cualquier caso práctico. Se mostró que es posible sustituir al muro de mampostería por tres elementos diagonales y obtener un comportamiento similar al original, tanto a nivel global como a nivel local. Es evidente que en etapas posteriores del desarrollo del presente modelo, y sobre todo, en su utilización práctica, el marco de concreto reforzado también debe ser sustituido por elementos barra, con comportamiento y parámetros mecánicos cuidadosamente seleccionados. 


\section{CONCLUSIONES}

El comportamiento de marcos con muros de relleno de mampostería sujetos a cargas laterales es muy complejo, con efectos no lineales prácticamente desde el inicio de la carga, modos de falla muy diversos, entre otras dificultades. Su modelado, aún con el método de los elementos finitos, no es sencillo, requiere tomar en cuenta muchos fenómenos que no son comúnmente incluidos en programas de análisis no lineal, tal es el caso de los elementos interfaz o modelos ortótropos de comportamiento. Se cuenta con muy poca información experimental de los parámetros necesarios para estos modelos, especialmente los que se refieren a la parte del ablandamiento por deformación, como son las energías de fractura en tensión y en compresión, el comportamiento ortótropo, adherencia entre mortero y piezas, así como entre mortero y concreto. En la literatura, aún la especializada, se habla muy poco y muy vagamente acerca de qué métodos de solución de sistemas no lineales son más adecuados a cada caso, y sobre las tolerancias recomendables para lograr ciertos niveles de precisión.

A pesar de estas dificultades, en este trabajo se demostró que, con base en la limitada información existente, es posible definir modelos numéricos basados en el método de los elementos finitos, como el presentado, capaces de reproducir satisfactoriamente el comportamiento de estas estructuras, al menos hasta los niveles de distorsión permitidos por los reglamentos de construcción. Es importante mencionar que, para explotar el potencial de este tipo de modelos numéricos, es necesario contar con información experimental adecuada que los respalde. El modelo de tres diagonales cuyas bases se propusieron en este trabajo tiene el potencial de representar las principales características del comportamiento, tanto global como local, de muros diafragma de mampostería. Una vez desarrollado completamente puede convertirse en una herramienta poderosa, de aplicación práctica, para el análisis y diseño de estructuras con este tipo de elementos.

\section{AGRADECIMIENTOS}

Se agradece el patrocinio de este trabajo por el Instituto Mexicano del Petróleo por medio del proyecto FIES "Desarrollo de modelos numéricos y herramientas de software para evaluar el proceso de fractura en estructuras de acero y concreto sometidas a cargas estáticas y/o cíclicas aplicando a la mecánica de fractura". El primer autor agradece al Consejo Nacional de Ciencia y Tecnología la beca que le otorgó para realizar sus estudios de maestría. 


\section{REFERENCIAS}

Combescure, D., Pegon, P. y Anthoine, A. (1995), "Modeling of the in-plane behaviour of masonry infilled frames", European Seismic Design Practice, Elnashai, A. S. (ed.), Balkema. Rotterdam, Holanda, pp. 621-629.

DDF (1996), "Normas técnicas complementarias para el diseño y construcción de estructuras de concreto." Gaceta Oficial del Departamento del Distrito Federal, México, D.F., marzo.

Lourenço, P.B. (1996), "Computational strategies for masonry structures", Tesis doctoral, Universidad Tecnológica de Delft, Holanda.

Mehrabi, A.B., Shing, P.B., Schuller, M.P. y Noland, J.L. (1994), "Performance of masonryinfilled R/C frames under in-plane lateral loads", Reporte CU/SR-94/6, Departamento de Ingeniería Civil, Ambiental y Arquitectónica, Universidad de Colorado en Boulder.

Mehrabi, A.B., Shing, P.B., Schuller, M., y Noland, J.L. (1996), "Experimental Evaluation of Masonry-Infilled R/C Frames," Journal of Structural Engineering, American Society of Civil Engineers, Vol. 122, No. 3, pp. 228-237.

Meli, R. (1979), “Comportamiento sísmico de muros de mampostería”, segunda edición, Informe No. 352, , Serie Azul, Instituto de Ingeniería, UNAM.

Meli R. y Salgado G. (1969), "Comportamiento de muros de mampostería sujetos a carga lateral”, Informe No 237, Serie Azul, Instituto de Ingeniería, UNAM.

Moghaddam, H.A. y Dowling, P.J. (1987), "The state of the art in infilled frames", Research Report No 87-2, Imperial College of Science and Technology, Londres, Gran Bretaña.

Orduña, A. (2000), "Modelo de muros de mampostería para el análisis no lineal de marcos de concreto reforzado con muros diafragma", Tesis de maestría, UNAM, México.

Rots, J.G. (1988), “Computational modeling of concrete fracture”, Tesis doctoral, Universidad Tecnológica de Delft, Holanda.

TNO (1996), "DIANA user's manual, release 6.1", TNO Building and Construction Research, Delft, Holanda. 